\title{
Evaluation of the constitutive expression levels of ch-TLR 3, ch-TLR 4, ch-TLR 15 and ch-TLR 21 genes in the Peripheral Blood Mononuclear Cells of native Indian poultry breeds, Aseel and Kadaknath
}

\author{
Meeta V. Patel ${ }^{1}$, Pankaj Kumar ${ }^{2}$, Hemen Das ${ }^{3}$, Abidali Dadawala $^{1}$, H. C. Chauhan ${ }^{4}$, B. S. Chandel $^{4}$,
} N. M. Shah ${ }^{1}$ and H. N. Kher

\begin{abstract}
1. Department of Veterinary Microbiology, Veterinary College, Sardarkrushinagar Dantiwada Agricultural University, Dantiwada - 385506, Gujarat, India; 2. Department of Veterinary Microbiology, Bihar Veterinary College, Patna, Bihar, India; 3. Department of Biochemistry and Physiology, Veterinary College, Sardarkrushinagar Dantiwada Agricultural University, Dantiwada - 385506, Gujarat, India; 4. Department of Animal Biotechnology, Veterinary College, Sardarkrushinagar Dantiwada Agricultural University, Dantiwada - 385506, Gujarat, India; 5. Registrar, Sardarkrushinagar Dantiwada Agricultural University, Dantiwada - 385506, Gujarat, India Corresponding author: Meeta V. Patel, email: mitapatel86@gmail.com
\end{abstract}

Received: 11-04-2013, Revised: 25-04-2013, Accepted: 25-04-2013, Published online: 14-06-2013

How to cite this article: Patel MV, Kumar P, Das H, Dadawala A, Chauhan HC, Chandel BS, Shah NM and Kher HN (2013) Evaluation of the constitutive expression levels of $c h-T L R 3, c h-T L R 4, c h-T L R 15$ and $c h-T L R 21$ genes in the peripheral blood mononuclear cells of native Indian poultry breeds, Aseel and Kadaknath, Vet World 6(8): 568-572, doi:10.5455/vetworld. 2013.568-572

\begin{abstract}
Aim: To assess the basal constitutive expression levels of ch-TLR3, ch-TLR 4, ch-TLR 15 and ch-TLR 21 in the peripheral blood mononuclear cells (PBMCs) in Aseel and Kadaknath chicks (Indian native poultry breeds) and to evaluate the differences in their general innate immune competence.

Materials and Methods: PBMCs were isolated from 21 day old Aseel and Kadaknath chicks $(\mathrm{n}=4)$ and were subjected to RNA isolation and cDNA synthesis. The basal expression of ch-TLR 3, 4, 15 and 21 was studied using real time PCR with SYBR green chemistry using 18 S-rRNA as the housekeeping gene.

Results: PBMCs isolated from Kadaknath chicks exhibited a significantly higher $(\mathrm{p}<0.05)$ constitutive expression of $c h$-TLR 3, ch-TLR 15 and ch-TLR 21 genes when compared to Aseel chicks. In comparison to Aseel, Kadaknath chicks recorded 14.774, 7.182 and 3.507 fold higher expressions of ch-TLR 3, ch-TLR 15 and $c h$-TLR 21 genes, respectively. In contrast, the constitutive expression of ch-TLR 4 was found to be higher (by 1.733 fold) in Aseel chicks.
\end{abstract}

Conclusion: Our results indicate that Kadaknath chicks are equipped with a better innate immune competence in comparison to Aseel chicks.

Keywords: Aseel, ch-TLR3, ch-TLR4, ch-TLR15, ch-TLR21, Kadaknath, Innate immune competence

\section{Introduction}

Innate arm of the immune system, once considered to play only a limited role in eliciting a protective immune response, has now acquired a centre stage and is now believed to play a pivotal role in the initiation as well as shaping the subsequent adaptive immune responses [1]. Cells of the innate arm are capable of recognizing a large number of invading pathogens using only a few germline encoded pathogen recognition receptors (PRRs), out of which the Toll like receptors (TLRs) are of prime importance [2]. TLRs recognise molecular patterns known as "Pathogen associated molecular patterns (PAMPs)" that are unique to microbes viz., Lipoteichoic acid (LTA), double stranded RNA, lipopolysaccharide (LPS), flagellin, unmethylated Cytosine triphosphate-phosphodiester link-Guanine triphosphate $(\mathrm{CpG})$ etc. which are recognised in chicken by $c h-T L R 2, c h-T L R$ 3, ch-TLR 4, ch-TLR 5 and ch-TLR 21 genes [3]. This pathogen sensing mechanism enables innate arm to recognise majority of the pathogens only with the help

This article is an open access article licensed under the terms of the Creative Commons Attribution License (http://creativecommons. org/licenses/by/2.0) which permits unrestricted use, distribution and reproduction in any medium, provided the work is properly cited. of a small repertoire of receptors. The efficiency of this system can be appreciated by the fact that recognition of LPS through TLR4 allows innate arm to recognise almost all of the gram negative bacteria. Interaction of these TLR ligands with the respective TLRs leads to either upregulation or downregulation of various immune related genes which have an important bearing on shaping subsequent adaptive responses into a more precise response [4].

Extensive breeding and selection programmes to improve economic attributes in commercial poultry flocks have compromised its innate immune competence in comparison to their native counterparts. The discoveries related to functioning of innate arm of the immune system have widely attracted the attention and infused a lot of interest in poultry scientists whose target is to improve the innate resistance of commercial flocks by looking for a better germplasm [5]. Generally, the immune competence of birds is assessed based on their level of complement activity or antibody response to Sheep RBCs (SRBCs). In the recent past, after the establishment of pivotal role of TLRs in pathogen recognition and generation of both innate and adaptive immune response, the constitutive and inducible expression of TLRs and other related genes has been correlated with general immune competence of the 
Table-1. List of primers used in the study

\begin{tabular}{|c|c|c|c|}
\hline Target Gene & Primer Sequence & Amplicon Size & Accession No \\
\hline ch-TLR 3 & $\begin{array}{l}\text { F: 5'-GTAAAG TGC CCC CTG TGC CGAA-3' } \\
\text { R: 5'-AGC TCG GTA CTC CCT GCG CT-3' }\end{array}$ & 135 & JF273967.1 \\
\hline ch-TLR 4 & $\begin{array}{l}\text { F: 5'-CCACCC TGGACT TGGACC TCA G-3' } \\
\text { R: 5'-AAG GCT GCTAGA CCC AGG TGC T-3' }\end{array}$ & 202 & NM_001030693.1 \\
\hline ch- TLR 15 & $\begin{array}{l}\text { F: 5'-ACCAGACAGACG GGG CTC GC-3' } \\
\text { R: 5'-CAAAGC GCCAGCATAAGC CCG-3' }\end{array}$ & 137 & NM_001037835.1 \\
\hline ch-TLR 21 & $\begin{array}{l}\text { F: 5'-TGA GGATGA TGGAGA CAG CGGAGA-3' } \\
\text { R: 5'-AGC GCG ATG GCATGT GGT GG-3' }\end{array}$ & 238 & NM_001030558.1 \\
\hline $18 S-r R N A$ & $\begin{array}{l}\text { F: 5'-GAT GCC GAC TCG CGATCC GG-3' } \\
\text { R: 5'-GCC GGG TGA GGT TTC CCG TG-3' }\end{array}$ & 186 & AF173612.1 \\
\hline
\end{tabular}

Table-2. Constitutive expression of $c h-T L R$ 3, ch-TLR 4, ch-TLR 15 and $c h-T L R 21$ in PBMCs from Aseel and Kadaknath chicks

\begin{tabular}{lllll}
\hline Parameters & \multicolumn{3}{c}{ Target genes } \\
\cline { 2 - 5 } & ch-TLR 3 & ch-TLR 4 & ch-TLR 15 & ch-TLR 21 \\
\hline Aseel chicks, Average delta Ct* values $(n=4)$ & $-14.97 \pm 0.44$ & $-10.169 \pm 0.30$ & $-9.944 \pm 0.51$ & $-13.018 \pm 0.46$ \\
Kadaknath chicks, Average delta Ct ${ }^{*}$ values $(n=4)$ & $-11.08 \pm 0.853$ & $-10.963 \pm 0.59$ & $-7.1 \pm 0.45$ & $-11.208 \pm 0.20$ \\
ddCt ${ }^{* *}$ in Kadaknath chicks in comparison to Aseel chicks & 3.885 & -0.794 & 2.844 & 1.81 \\
Level of relative expression in Kadaknath chicks in & 14.774 & -1.733 & 7.182 & 3.507 \\
comparison to Aseel chicks & & & \\
\hline
\end{tabular}

$*$ delta $\mathrm{Ct}=\mathrm{Ct}$ of housekeeping gene-Ct of Target gene

$* * \mathrm{dd} \mathrm{Ct}=$ delta Ct of target gene (Kadaknath chicks) - delta Ct of target gene (Aseel chicks)

Relative expression was calculated using IF command in excel $=\operatorname{IF}\left(\mathrm{ddCt}>0,2^{\wedge} \mathrm{ddCt},-\left(2^{\wedge}-\mathrm{ddCt}\right)\right.$

fowl [6]. A better disease tolerance of Aseel and Kadaknath, Indian chicken breeds, in comparison to their commercial counterparts is well documented $[7,8]$. Nevertheless, majority of the studies that were aimed at determining the immune competence, on the basis of constitutive and inducible expression of chTLRs and other related genes, in fowls were conducted in commercial breeds and the knowledge related to the expression of these innate immune markers in native poultry breeds such as Aseel and Kadaknath is largely unknown.

Thus, we designed this study with an objective to evaluate the constitutive expression of genes that encode four important chicken TLRs i.e., ch-TLR 3, ch-TLR 4, ch-TLR 15 and ch-TLR 21 in the peripheral blood mononuclear cells (PBMCs) of Indian native breeds; Aseel and Kadaknath.

\section{Materials and Methods}

Ethical approval: This study was conducted as per the provisions of the Institute Animal Ethics Committee.

Chicks: Day old chicks belonging to Aseel and Kadaknath breeds were purchased from the Central Poultry Research Station, Anand. Chicks were maintained in the Poultry Demonstration Unit, Veterinary College, SDAU-Sardarkrushinagar under standard managemental procedures and during the entire experimental period they were provided with ad-libitum feed and water.

Sample collection: Approximately $1.5 \mathrm{ml}$ of blood from four chicks of either sex from Aseel and Kadaknath breeds was collected aseptically from the jugular vein into heparin coated vials on the $21^{\text {th }}$ day of their age.

Peripheral blood mononuclear cells (PBMCs) isolation: Individual blood samples were mixed with PBS $(1: 1$ dilution), $3 \mathrm{ml}$ of this blood - PBS mixture was then layered carefully onto $3 \mathrm{ml}$ of Histopaque (1.077,
sigma-Aldrich) and centrifuged at $1800 \mathrm{rpm}$ for 20 minutes in swing out rotors. White ring at the interface containing the mononuclear cells/PBMCs was carefully aspirated and washed twice with HBSS followed by centrifugation at $800-1000 \mathrm{rpm}$ for 10 minutes.

Relative expression study: PBMCs@2X106 were subjected to RNA isolation, c-DNA preparation and relative quantification using real time PCR (ABI, 7500). The primers used in the study were designed using the softwares available online (Table-1). The primers were of small amplicon size (100-300 bp), amplified at $58^{\circ} \mathrm{C}$ and showed primer efficiency in the range of 1.8-2.0. 18S rRNA was considered as the housekeeping gene for this study. The reaction conditions were standardized with SYBR green chemistry and all the primers produced a single peak in the dissociation curve analysis confirming the high specificity of the products. To determine the relative expression levels of genes investigated between Aseel and Kadaknath breeds, the constitutive expression in PBMCs from Aseel breed was considered as a unit (control) for respective genes under comparison.

Statistical analysis: Data were analysed using WASP 1 software available online in the statistical package at ICAR-Goa complex site (http:/www.icargoa.res.in/ wasp/rbd1.php).

\section{Results}

PBMCs isolated from both the Indian native breeds were found to have constitutive expression of ch-TLR 3, ch-TLR 4, ch-TLR 15 and ch-TLR 21. In comparison to Aseel chicks, PBMCs from Kadaknath chicks showed significantly higher $(p<0.05)$ constitutive expression of ch-TLR 3, ch-TLR 15 and ch$T L R 21$. Considering unit expression of $c h-T L R$ genes 
investigated here in Aseel chicks, Kadaknath chicks recorded $14.774,7.182$ and 3.507 fold higher expressions of ch-TLR 3, ch-TLR 15 and ch-TLR 21 genes, respectively. However, an exception was that the constitutive expression level of ch-TLR 4 was found to be apparently higher in PBMCs isolated from Aseel chicks than that of Kadaknath chicks. Though non-significant, the expression of TLR 4 was found to be 1.733 times higher in Aseel than Kadaknath chicks PBMCs (Table-2).

\section{Discussion}

The discovery of toll-like receptors (TLRs), particularly on the cells of innate arm of immune system $[9,10]$ and their critical role in the providing immunity has aroused intense curiosity in the scientific arena and the studies conducted in the recent years on this subject has greatly improved our understanding of the functioning of immune system [11]. A better understanding of innate arm could also assist poultry scientists to meticulously look for desired immunerelated genes with a potential to be incorporated in marker assisted selective breeding programmes. Keeping these facts in mind, this study was conducted on two Indian native breeds of fowl i.e., Aseel and Kadaknath which are reported to have enhanced abilities to fight infectious diseases when compared to abilities of the commercial breeds $[12,13]$.

TLRs are a group of highly conserved and germline encoded pattern recognition receptors. Chicken genome has orthologs of mammalian TLR 1, TLR 2, TLR 3, TLR 4, TLR 5 and TLR 7 whereas orthologs of $T L R$ 8, TLR9 and TLR 10 appears to be either defective or missing [14]. TLR 3 recognises double stranded RNA intermediate formed during viral replication phase and directs the immune system to mount an antiviral response [15]. TLR 4 recognises LPS from gram negative bacteria [16]. An avian orthologue of mammalian TLR 4 was characterized [17]. TLR 15 was discovered in chicken and considered to be an avian specific member of the TLR family. The role of TLR 15 in innate defence against Salmonella Enterica serovar Typhimurium is suggested [18]. Mammals recognise and respond to $\mathrm{CpG}$ ODN through TLR 9 which was found missing in the chicken genome. However, chicken showed somewhat similar responsiveness to CpG ODN. Later on, the recognition of CpG ODN was reported to be mediated by TLR 21 [19]. The role of TLR 3, TLR 4, TLR 15 and TLR 21 in host defense against viral and bacterial pathogens is well documented [20-23]. These earlier findings formed the premise for selecting ch-TLR 3, ch-TLR 4, ch-TLR 15 and ch-TLR 21 genes and to study their constitutive expression in PBMCs of Indian native Aseel and Kadaknath breeds which enables us to better under-stand the general immune competence of the native Indian poultry breeds. The PBMCs were collected from 21 day old chicks which coincide with the maturation of bursa at three weeks of their age and thereby lead to an acquisition of better immune competence.

In the present study, PBMCs isolated from 21 day old Kadaknath chicks had significantly higher $c h-T L R$ 3, ch-TLR 15 and ch-TLR 21 m-RNA transcript expression, whereas ch-TLR 4 had apparently higher expression in Aseel. Differential expression of various important immune-related genes has been correlated with the disease susceptibility or resistance pattern of the host [24]. The difference in ch-TLR 15 gene expression by heterophils of lines A (Salmonella resistant) and B (Salmonella susceptible) is speculated to account for some of the observed differences between the lines in their susceptibility to infection [25]. The bacterial load in susceptible chickens was significantly higher than that in the resistant chickens and ch-TLR4, ch-TLR2-1 and ch-TLR21 expression was highly diminished in the leukocytes of susceptible chickens when compared with those of resistant chickens [26]. Higher expression of ch-TLR 4, ch-TLR 15, ch-TLR 21 and a few other genes involved with TLR activation pathways was reported with Salmonella Enteritidis (SE) infection in chicken line A (resistant to Salmonella Enteritidis infection) [27]. The significantly higher expression of ch-TLR3, ch-TLR 15 and ch-TLR 21 in Kadaknath chicks, observed in the present study, is suggestive of a better immune competence of Kadaknath in comparison to Aseel chicks. In present study, constitutive expression of ch-TLR 4 in PBMCs from Aseel chicks was found to be apparently higher than that of Kadaknath chicks. Contrary to our reports, significantly higher expression of ch-TLR 4 and ch-TLR 5 in heterophils from Kadaknath than in heterophils of Aseel, Necked neck, Dwarf and White Leghorn lines was reported earlier [28]. Contrasting observations in the expression level of ch-TLR4 in the previous and our current reports might be due to difference in the target cells used in the two studies i.e., heterophils in the earlier study and PBMCs in our study. Nevertheless, these reports indicate a differential expression of TLRs in different immune compartments.

Poultry health is constantly under the threat of a wide array of pathogens of which viral and bacterial diseases have most devastating impact. The better immune competence in indigenous chicken is ascribed to higher complement activity, higher serum lysozyme level and antibody response $[13,29]$. The basal TLR mRNA expression profiles in different cells and tissues are suggestive of an individual's ability in responding to a challenge [30]. The present study indicates a better immune competence of Kadaknath breed to deal with a variety of the invading pathogens. In this context, it is important to note that we have not used any infectious model to ascertain the immune competence of these two breeds by exposing them to pathogenic challenge. Thus, ingenious experimental designs combined with the efficiency of latest tools and techniques are required to test this notion further.

\section{Conclusion}

Higher expression of $T L R$ genes has been 
correlated with enhanced ability of the host to encounter invading pathogens. Thus, findings from the present study reflect a better immune competence of Kadaknath over Aseel chicks. However, considering the operational complexity of the immune response, further investigations are needed to fully ascertain how these differences in Aseel and Kadaknath chicks translate into development of an efficacious adaptive immune response. Future studies on Aseel and Kadaknath chicks along with other native breeds with target tissues like spleen, bursa, proximal GI tract, caecum etc. is highly warranted which will be of immense help in identifying important alleles involved in deciding resistance or susceptibility patterns of the host. We strongly believe that these future studies will pave way for the incorporation of better genetic resources in designing selective breeding programmes for efficient control and prevention of diseases.

\section{Authors' contributions}

All authors contributed equally. All authors read and approved the final manuscript.

\section{Acknowledgments}

Authors are thankful to Dr. A. K. Jain, Professor and Head, Department of LPM for providing facilities to maintain the experimental birds in the Poultry Demonstration Unit. Thanks are also extended to the Dean, College of Veterinary science and $\mathrm{AH}$ and to the Director of Research, SDAU, Sardarkrushinagar for their extensive support which lead to an uninterrupted conduct of this study. Financial assistance received from SDAU, Sardarkrushinagar and Department of Biotechnology, Government of India, New Delhi is duly acknowledged.

\section{Competing interests}

The authors declare that they have no competing interest.

\section{References}

1. Kumar, H., Kawai, T. and Akira, S. (2009) Toll-like receptors and innate immunity. Biochem Biophys Res Commun., 388(4):621-625.

2. Kumar, H., Kawai, T. and Akira, S. (2011) Pathogen recognition by the innate immune system. Int Rev Immunol., 30(1):16-34.

3. Paula, M. S., Brisbina, J. T., Abdul-Careemb, M. F. and Sharif, S. (2013) Immunostimulatory properties of Toll-like receptor ligands in chickens. Vet. Immunol. Immunopathol. 152:191-199.

4. Van, D. D., Medzhitov, R. and Shaw, A. C. (2006) Triggering TLR signaling in vaccination. Trends Immunol., 27(1):49-55.

5. Swaggerty, C. L., Pevzner, I. Y., He, H., Genovese, K. J., Nisbet, D. J., Kaiser, P. and Kogut, M. H. (2009) Selection of broilers with improved innate immune responsiveness to reduce on-farm infection by foodborne pathogens. Foodborne Pathog Dis. 6(7):777-83.

6. Abasht, B., Kaiser, M.G., Van, P.J. and Lamont, S.J. (2009) Genetic lines differ in Toll-like receptor gene expression in spleens of chicks inoculated with Salmonella enterica serovar Enteritidis. Poult Sci, 88 (4):744-9.

7. Rout, P. K., Pani, P. K. and Naithani, S. (1992) Genetic susceptibility of indigenous chicks to subgroup A Rous sarcoma virus inoculated via the chorioallantoic membrane.
Vet. Immunol. Immunopathol., 33(1-2):89-102.

8. Wimmers, K., Ponsuksili, S., Hardge, T., Valle-Zarate, A., Mathur, P. K. and Horst, P. (2000) Genetic distinctness of African, Asian and South American local chickens. Anim. Genet., 31:159-165.

9. Applequist, S. E., Wallin, R. P. and Ljunggren, H. G. (2002)Variable expression of Toll-like receptor in murine innate and adaptive immune cell lines. Int. Immunol., 14 (9): 1065-74.

10. Iqbal, M., Philbin, V. J. and Smith A. L. (2005) Vet. Immunol.Immunopathol., 104(1-2):117-27.

11. St Paul, M., Paolucci, S., Read, L. R. and Sharif, S. (2012) Characterization of responses elicited by Toll-like receptor agonists in cells of the bursa of Fabricius in chickens. Vet Immunol Immunopathol.; 149(3-4): 237-44.

12. Rout, P. K., Pani, P. K. and Naithani, S. (1992) Genetic susceptibility of indigenous chicks to subgroup A Rous sarcoma virus inoculated via the chorioallantoic membrane. Vet. Immunol. Immunopathol. 33(1-2):89-102.

13. Kundu, A., Singh, D. P., Mohapatra, S. C., Dash, B. B., Moudgal, R. P. and Bisht, G. S. (1999) Antibody response to sheep erythrocytes in Indian native vis-à-vis imported breeds of chickens. Br. Poult. Sci., 40:40-43.

14. Schwarz, H., Schneider, K., Ohnemus, A., Lavric, M., Kothlow, S., Bauer, S., Kaspers, B. and Staeheli, P. (2007) Chicken toll-like receptor 3 recognises its cognate ligand when ectopically expressed in human cells. J Interferon Cytokine Res. 27(2):97-101.

15. Akira, S. and Hemmi, H. (2003) Recognition of pathogenassociated molecular patterns by TLR family. Immunol Lett., 85(2):85-95.

16. Akira, S., Uematsu, S. and Takeuchi, O. (2006) Pathogen recognition and innate immunity. Cell 24; 124(4): 783-801.

17. Leveque, G., Forgetta, V., Morroll, S., Smith, A. L., Bumstead, N., Barrow, P., Loredo-Osti, J. C., Morgan, K., Malo, D. (2003) Allelic variation in TLR4 is linked to susceptibility to Salmonella enterica serovar Typhimurium infection in chickens. Infect Immun. 71(3): 1116-24.

18. Higgs, R., Cormican, P., Cahalane, S., Allan, B., Lloyd, A. T., Meade, K., James, T., Lynn, D. J., Babiuk, L. A. and O'farrelly, C. (2006) Induction of a novel chicken Toll-like receptor following Salmonella enterica serovar Typhimurium infection. Infect Immun., 74(3):1692-8.

19. Brownlie, R., Zhu, J., Allan, B., Mutwiri, G. K., Babiuk, L. A., Potter, A. and Griebel, P. (2009) Chicken TLR21 acts as a functional homologue to mammalian TLR9 in the recognition of $\mathrm{CpG}$ oligodeoxynucleotides. Mol Immunol., 46(15):3163-70.

20. Lu, Y., Sarson, A.J., Gong, J., Zhou, H., Zhu, W., Kang, Z., Yu, H., Sharif, S. and Han, Y. (2009) Expression profiles of genes in Toll-like receptor-mediated signaling of broilers infected with Clostridium perfringens. Clinical and Vaccine Immunology, 16:1639-1647.

21. MacKinnon, K.M., He, H., Nerren, J.R., Swaggerty, C.L., Genovese, K.J. and Kogut, M.H. (2009) Expression profile of toll-like receptors within the gastrointestinal tract of 2day-old Salmonella enteriditis-infected broiler chickens. Vet. Microbiol.,137(3-4):313-9.

22. Nang, N.T., Lee, J.S., Song, B.M., Kang, Y.M., Kim, H.S., Seo, S.H. (2011) Induction of inflammatory cytokines and toll-like receptors in chickens infected with avian H9N2 influenza virus. Vet. Res., 18;42(1):64.

23. Nerren, J.R., Swaggerty, C.L., MacKinnon, K.M., Genovese, K.J., He, H., Pevzner, I. and Kogut, M.H.(2009) Differential mRNA expression of the avian-specific toll-like receptor 15 between heterophils from Salmonellasusceptible and -resistant chickens. Immunogenetics, 61(1):71-7.

24. Adams, S. C., Xing, Z., Li, J. and Cardona, C.J. (2009) Immune-related gene expression in response to H11N9 low pathogenic avian influenza virus infection in chicken and Pekin duck peripheral blood mononuclear cells. Mol. 
Immunol., 46(8-9):1744-9.

25. Nerren, J. R., Swaggerty, C. L., MacKinnon, K. M., Genovese, K. J., He, H., Pevzner, I. and Kogut, M. H.(2009) Differential mRNA expression of the avian-specific toll-like receptor 15 between heterophils from Salmonellasusceptible and -resistant chickens. Immunogenetics, 61(1): 71-7.

26. Gou, Z., Liu, R., Zhao, G., Zheng, M., Li, P., Wang, H., Zhu, Y., Chen, J. and Wen, J. (2012) Epigenetic modification of TLRs in leukocytes is associated with increased susceptibility to Salmonella enteritidis in chickens. PLoSOne, 7(3): e33627.

27. Kogut, M. H., Chiang, H. I., Swaggerty, C. L., Pevzner, I. Y. and Zhou, H. (2012) Gene Expression Analysis of Toll-Like Receptor Pathways in Heterophils from Genetic Chicken Lines that Differ in Their Susceptibility to Salmonella enteritidis. Front Genet. 3:121.
28. Ramasamy, K. T., Reddy, M. R., Raveendranathan, D. N., Murugesan, S., Chatterjee, R. N., Ullengala, R.and Haunshi, S. (2010) Differential expression of Toll-like receptor mRNA in White Leghorn and indigenous chicken of India. Vet. Res. Commun., 34(7):633-9.

29. Baelmans, R., Parmentier, H. K., Nieuwland, M. G., Dorny, P., Demey, F. and Berkvens, D. (2005) Haemolytic complement activity and humoral immune responses to sheep red blood cells in indigenous chickens and in eight German Dahlem Red chicken lines with different combinations of major genes (dwarf, naked neck and frizzled) of tropical interest. Trop. Anim. Health Prod., 37:173-186.

30. Tirumurugaan, K. G., Dhanasekaran, S., Dhinakar, R. G., Raja, A., Kumanan, K. and Ramaswamy, V. (2010) Differential expression of toll-like receptor mRNA in selected tissues of goat (Capra hircus). Vet. Immunol. Immunopathol., 133: 296-301.

$* * * * * * * *$ 